CONEXIONES LATINOAMERICANAS A INTERNET:

\title{
Ventanas al mundo
}

\section{Eliza Tanner}

Máster en comunicación de masas, Escuela de Periodismo y Telecomunicaciones Walter Cronkite, Universidad Estatal de

Arizona. Doctora (c) en periodismo por la Escuela de

Periodismo y Comunicación de Masas, Universidad de

Wisconsin-Madison. [eatanner@students.wisc.edu]

Mientras que Chile, Brasil y México son líderes en número de computadores y conexiones a Internet, otros países de la región -especialmente en Centroamérica- aún no logran siquiera las exigencias técnicas mínimas para establecer comunicaciones fluidas. Y aunque en la mayoría de los países la tendencia es a privatizar las telecomunicaciones, en otros el Estado se ha arrogado la facultad de controlar las conexiones. Sin embargo, se aprecia una característica común, sostiene Eliza Tanner: el uso que se le está dando a la red en América Latina responde muchas veces al intento de solucionar problemas regionales, como el hambre o las amenazas al medioambiente, lo que pone en duda la tesis de un flujo unidireccional desde el Norte.

- n una montaña del sur de México, el subcomandante zapatista Marcos prepara un comunicado en que denuncia al gobierno: "A la gente de México, a la gente de los gobiernos del mundo, a la prensa nacional e internacional", escribe en su computador portátil, conectado al encendedor de cigarrillos de un camión. Cuando termina, los correos contrabandean los discos a los simpatizantes en la ciudad. De ahí, los partidarios colocan la información en Internet y en segundos la gente en todo el mundo puede leer el último mensaje de los rebeldes en Chiapas. "Cuando recientemente la policía atacó por sorpresa los escondites en el estado sureño de Veracruz, se informó que capturaron más discos de computador que balas ${ }^{1}$. En 


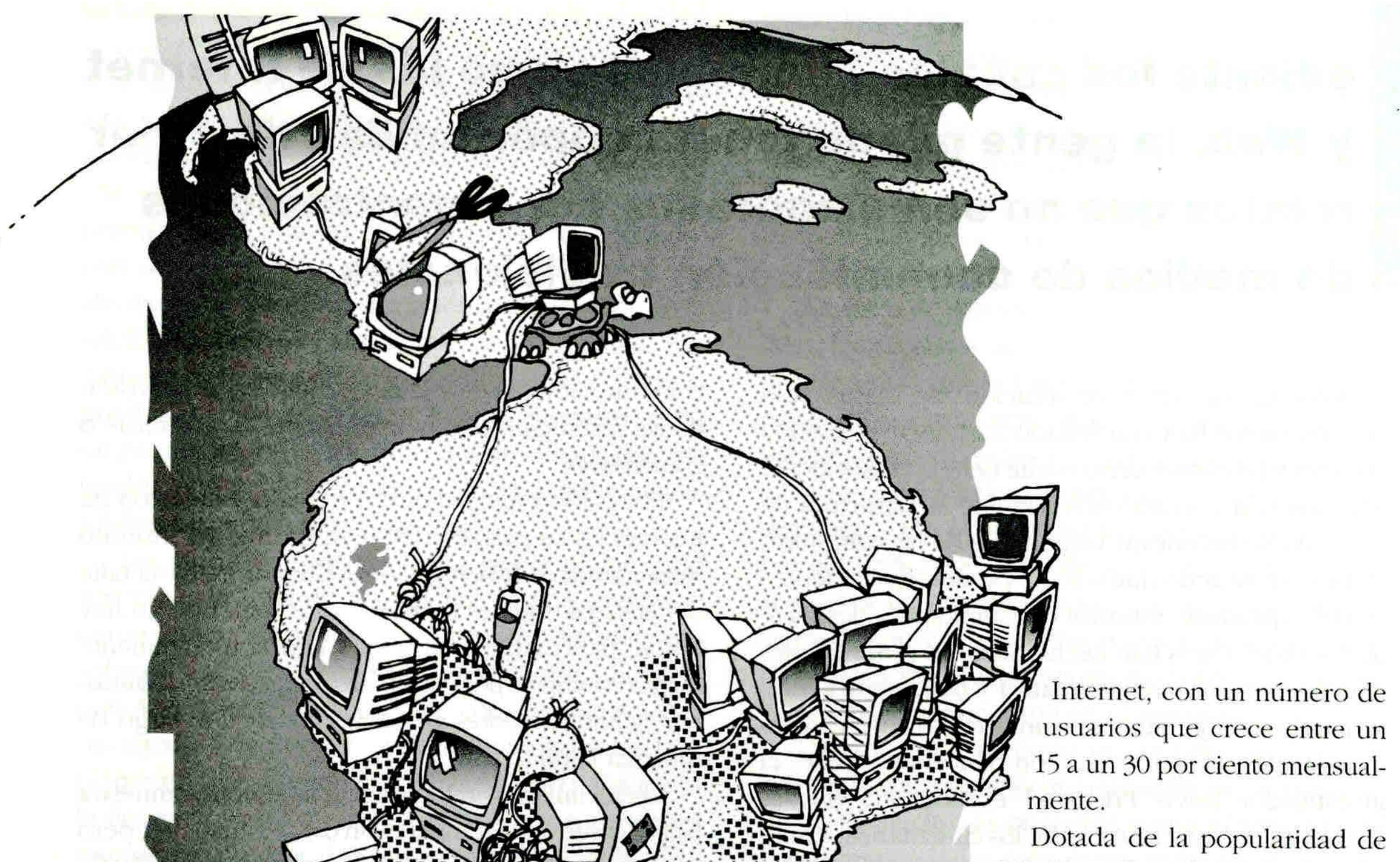
World Wide Web, Internet se ha difundido en los últimos dos años introduciendo temas que incluyen política, negocios, medios de información.

A mediados de 1996, un catálogo en línea muy importante se jactaba de haber registrado 30 millo-

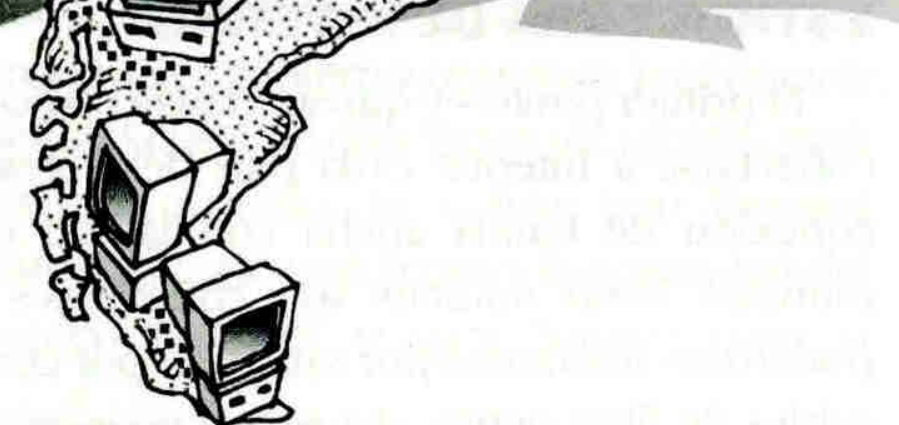

el sur del continente, la oficina chilena de Greenpeace envía un correo electrónico al grupo peruano de organizaciones no gubernamentales (ONGs), donde advierte que el barco croata Triglav va a descargar desechos tóxicos a lo largo de las costas de Sudamérica. Puestas sobre aviso, las ONGs se movilizan, coordinan sus protestas y persuaden al gobierno para prohibir al barco atracar en Perú. En todas partes de América Latina, individuos y grupos se conectan a nes de páginas Web que correspondían a 275.600 servidores alrededor del mundo. Aunque el crecimiento se ha inclinado fuertemente (alrededor de un 94\% de todos los computadores anfitriones están ubicados en Norteamérica, Europa, Australia y Japón), los países en vías de desarrollado también establecen comunicaciones en Internet. De los países latinoamericanos, Brasil, México, Chile y Argentina aumentaron sus conexiones a principios de 1990. Otros, como Bolivia y Nicaragua, sólo consiguieron conectarse a Internet desde mediados de 1995.

El propósito de este artículo es examinar algunas consecuencias de las comunicaciones a través de Internet en América Latina, su uso y su estructura.
1. DOYLE, LEONARD: Rebels try to advance via Internet", The Independent, 7 de marzo de 1995 , p. 11. Véanse también los comunicados del EZLN en http:// www.peak.org/ justin ezln/communiques.html; información sobre Chiapas, en http:// lanic.utexas.edu/la/ mexico/chiapas.html, y otras páginas relacionadas de la Web. 


\section{ediante los canales informales como los de Internet y Web, la gente puede tener la oportunidad de crear relatos que no son elaborados por organizaciones de medios de comunicación tradicionales.}

2. MCCHESNEY, ROBERT: "The Internet and U.S. Communication PolicyMaking in Historical and Critical Perspectiven, Journal of Communication 46 (1996), p. 99.

3. FRIFDLAND, LEWIS: Electronic democracy and the new citizenship", Media, Culture \& Society 18 (1996), p. 207

4. RIVERO, JUAN [juanr@stat.ivic.ve], "Re: Preguntas sobre la WWW, email a la autora, 12 de marzo de 1996.

5. QUTTTNER, JOSHUA: Wiring the World, Time,

17 de julio de 1995 .

p. 52 .
Especialistas en comunicación de masas sólo recientemente han comenzado a estudiar el sistema Internet y las consecuencias que tiene en la sociedad. Algunos han criticado severamente la estructura de mercado de las nuevas tecnologías de las comunicaciones asegurando que son un "producto característico del capitalismo mundial que aumenta la desigualdad social ${ }^{2}$. Otros han hecho notar las posibilidades para la comunicación alternativa y democrática, presentando sus argumentos en un sistema de movimientos sociales y de comunicación para el desarrollo. El investigador Lewis Friedland escribe que nuevas formas de comunicación entre los ciudadanos, como las que ofrece Internet, pueden "desafiar y profundizar los conceptos democráticos deliberativos" ${ }^{3}$.

Muchos países han creado estructuras duales de comunicación donde las formas alternativas existen junto con los medios de comunicación masivos tradicionales, "occidentales". En algunos lugares, las sociedades adoptan tecnologías de los medios de comunicación extranjeros y los usan para diferentes propósitos, algunas veces radicales o revolucionarios. Teóricamente, Internet es un medio de comunicación cuya tecnología puede utilizarse para una comunicación horizontal, descentralizada y "alternativa".

Estos modelos alternativos de comunicación se idearon, en parte, como una reacción contra las estructuras tradicionales de los medios de comunicación masivos, dentro de los países y también en el extranjero.

Con los canales informales de comunicación como los proporcionados por Internet y $\mathrm{Web}$, la gente puede tener la oportunidad de crear relatos que no son elaborados por organizaciones de medios de comunicación tradicionales. En algunos casos, la comunicación puede incluso escapar a la supervisión de las instituciones gubernamentales, académicas o comerciales.

El desafío principal que enfrenta la expansión de Internet en su país, escribe Juan Rivero, del Instituto Venezolano de Investigaciones Científicas, es "la falta de recursos. Que es lo mismo que decir que no hay fondos para nada " ${ }^{4}$. Esta queja surgió constantemente en mis contactos por correo electrónico con webmasters (administradores de páginas Web) a lo largo de América Latina.

El crecimiento de Internet en la región se muestra bajo cuando se lo compara con datos mundiales, pero las cifras varían considerablemente según el país. En esta sección, examinaré la estructura física de Internet en América Latina, los desafíos que enfrentan los países en la creación de la nueva tecnología de comunicación y los puntos de acceso.

\section{ESTRUCTURA DE INTERNET}

El primer punto -y quizás el más obvio- es que al conectarse a Internet cada país debe gestionar una conexión de banda ancha con la red de alcance mundial. Estas uniones son conexiones rápidas y poderosas realizadas por satélite o por conductos de cables de fibra óptica. "La nueva economía de información puede quizás redistribuir la riqueza de las naciones, pero una cosa es segura: para obtener un cupo, cualquier país necesita una red de banda ancha" ${ }^{5}$. Las primeras instituciones en crear redes en los países de América Latina son generalmente universidades y el gobierno. Más recientemente, empresas internacionales están adaptando sus propias conexiones al sistema Internet. La red de alcance mundial es una conexión de muchas redes de menor 
tamaño que se clasifican de acuerdo con la velocidad y con el tipo de hardware. La velocidad de la conexión es crucial. "Las diferencias entre el acceso de alta y baja velocidad de acceso (high y low-end access) se pueden ilustrar al comparar a un investigador en Estados Unidos, que tiene una conexión permanente a una red multi-megabit por segundo, con un investigador en África, que se conecta a alrededor de 200 caracteres por segundo en una línea telefónica inestable. Una consecuencia de estos números es que los académicos de los Estados Unidos pueden ir a buscar un texto de gran envergadura en un par de segundos, mientras que su colega africano tendría que gastar todo el día acumulando cuentas telefónicas para hacer lo mismo" 6 .

Los países en América Latina han implementado servicios de Internet en muchas formas. Algunos, como Chile, casi no tienen supervisión del gobierno sobre el crecimiento comercial de Internet, mientras que Argentina y Brasil cuentan con comunicaciones nacionales abiertas, pero también con algunas restricciones en el acceso internacional. México y Venezuela tienen autorización para los servicios comerciales internacionales basados en Internet. En abril de 1996 el gobierno de Guatemala decretó ilegales las conexiones a Internet a través de sistemas de telecomunicaciones o satélites privados, y dispuso que sólo se puede tener acceso directamente a Internet a través de la compañía estatal Guatel. "El reglamento de Guatel contiene un lenguaje muy vago que permitiría efectivamente que el gobierno censurara las comunicaciones. Según la lista de conductas prohibidas para los usuarios, por ejemplo, se incluye usar Internet para propósitos que atenten "contra la seguridad del Estado y orden público" o para "causar daño o perjuicio al Estado" " 7 . Sin embargo, no se sabe exactamente con claridad qué efectos tendrá este reglamento, en especial, para los proveedores locales que ya tienen uniones satelitales a Internet. En América Latina sólo unos pocos países (Cuba, Guyana, Belice, Haití y Paraguay) aún no tienen acceso completo a Internet, aunque esto tendría que cambiar a finales de este año.

Analistas de negocios alaban la estabilidad económica, los procesos de privatización, el respaldo a las inversiones y las reformas administrativas en los países de América Latina. Brasil, Colombia y Chile son los mercados de redes de más rápido crecimiento, mientras que México, Argentina y Venezuela han disminuido el suyo debido a los problemas económicos en 1995. Argentina, Brasil y Chile abrieron sus secciones de Internet a los usuarios comerciales a mediados de 1995. Grandes compañías de computación están extendiendo sus negocios a América Latina y esperan ganar un cupo en el mercado.

Un país que a menudo se cita como ejemplo de esta expansión de telecomunicación es, justamente, Chile. De hecho, es conocido como el mercado de telecomunicaciones más competitivo en el mundo; también, como un "campo de experimentación" para la fibra óptica, los teléfonos personales, los conmutadores digitales y otros avances en telecomunicaciones. El proceso de privatización de la industria chilena de telecomunicaciones comenzó en 1978 y ha experimentado una tasa media de crecimiento anual de 19,5\% desde 1990. La Compañía de Teléfonos de Chile S.A. (CTC) y la Empresa Nacional de Telecomunicaciones (Entel) se privatizaron en 1988, y en 1994 las empresas pudieron tener igual acceso a la competencia por el servicio de larga distancia nacional e
6. Holderness, Mike: "Falling through the net; developing nations lack Internet accessn, New Statesman \& Society, 13 de octubre de 1995 ,

p. 24

7. "Monopoly on Internet in Guatemala", http:// www.guatered.com/ news/gweekly.htm, Guatemala Weekly, 2026 abril de 1996

\section{rasil es uno de los mercados computacionales con el
crecimiento más rápido del mundo, con el $40 \%$ de todas
las ventas del continente; Chile, por su parte, presenta
el cien por ciento de sus redes telefónicas digitalizadas.}




\section{I contrario de Chile, Brasil y México, la mayoría de los países de Centroamérica se han conectado recientemente a Intermet. Dar este paso significa también enfrentar una gran cantidad de desafíos.}

internacional. Chile presenta un $100 \%$ de redes de teléfonos locales digitalizados y con pocas regulaciones por parte de gobierno. En este país las tarifas de llamadas de larga distancia son las más baratas en el mundo desde que cuenta con un sistema multiportador que permite que los usuarios elijan cualquier compañía cada vez que hacen un llamado. El proveedor del servicio Internet más grande es la Red Universitaria Nacional (Reuna), la que sirve a cerca de un $80 \%$ de los usuarios de Chile. No obstante, la competencia es intensa, ya que existen otras 16 compañías que ofrecen conexiones a Internet.

Brasil es uno de los mercados computacionales con el crecimiento más rápido del mundo, con el $40 \%$ de todas las ventas en América Latina. Con sus cambios en las regulaciones gubernamentales, Brasil está tratando de modelar su sistema de telecomunicaciones a la luz de los planes de los Estados Unidos y de Chile. A fines de 1995 el sector privado reemplazó el sistema de Embratel (Empresa brasileña de telecomunicaciones) y de Telebras (Telecomunicaciones brasileñas) en el suministro de los servicios comerciales de Internet. Sin embargo, el gobierno brasileño a través de la Rede nacional de pesquisa (RNP), mantiene la determinación de continuar con el servicio de

8. MACDONALD, CHRISTINE: "Mexico Steering Toward The Info Superhighway", San Francisco Chronicle, 6 de marzo de 1995, p. D1

9. DEI. CARPIO, ABEL. [webmast@wari.rcp.net.pe], "Re: Preguntas sobre la

WWW", email a la autora, 18 de marzo de 1996. a Internet de la UNAM es la más antigua en el país; los estudiantes y profesores pueden conectarse sin cargo desde el campus o desde sus casas. La UNAM puede costear los gastos de operación al vender sus servicios a otras universidades y bancos mexicanos. Sin embargo, en la mayor parte del México antiguo, los servicios de teléfono costosos disminuyen el crecimiento de Internet ${ }^{8}$.

Al contrario de Chile, Brasil y México, la mayoría de los países de Centroamérica se han conectado recientemente a Internet. Dar este paso significa también enfrentar una gran cantidad de desafíos.

\section{LOS DESAFIOS}

Además de los conflictos en el propósito y en el uso de Internet, los desafíos para difundirla incluyen las regulaciones del gobierno, los altos costos de acceso debido a los elevados cargos de los teléfonos locales, y a que la información en Internet se encuentra en su gran mayoría en inglés. En Perú, la Red Científico Peruana $(R C P)$ es el único proveedor local que permite acceder a Internet. Existen muchos desafíos, escribe Abel del Carpio, webmaster de la $R C P$. "El primero es expandir nuestra conexión de ancho de banda, alcanzar a más peruanos, interconectar puntos en provincias y departamentos, aumentar la utilidad de Internet; en resumen: promover el desarrollo de las telecomunicaciones en el ámbito nacional y en el internacional"?.

José Soriano, fundador del $R C P$, dice: "Aquí la gente comienza a entender que la información es una forma de riqueza y que Internet representa información. Es una forma de ponerse al día con el resto del mundo". Uno de los mayores desafíos es el costo; el acceso individual a Internet tiene un costo en la $R C P$ 
de 37 dólares al mes, lo que no incluye el cargo del teléfono, ni del computador. Además, la mayoría de los usuarios de Internet usa el computador de otra persona. En mayo de 1995, Perú tenía 10.000 cuentas de Internet, pero sólo 550 computadores conecta$\operatorname{dos}^{10}$.

El mayor desafío que enfrenta el crecimiento de Internet en Bolivia es la carencia de recursos, escribe Hubert Abasto Revilla. Por ejemplo, a las organizaciones de Internet les toma meses para garantizar las líneas telefónicas, la gente encargada de las telecomunicaciones no entiende la importancia de Internet, las organizaciones ven a estos negocios como amenazas para sus monopolios, los teléfonos son de baja calidad y el equipo es malo en algunos lugares", escribe. “También existe un problema económico que no nos permite contar con los medios necesarios para una mejor conexión", señala Abasto ${ }^{11}$.

El mismo problema existe en Nicaragua. La webmaster Yadira Mena dice que un desafío es la conexión "de escaso ancho de banda que tenemos con Internet ( $64 \mathrm{Kbps}$ ) y los múltiples problemas al enviar [los datos] al extranjero, que en muchas ocasiones nos dejan fuera del aire o nos desconectan" 12 . Un webmaster universitario de Nicaragua coincide: "Tenemos problemas con la velocidad de las comunicaciones. Este conflicto es el resultado de la infraestructura de comunicaciones del país. Todos los computadores conectados a Internet tienen este problema. Tres países centroamericanos comparten la unión satelital y muchas veces el tráfico es demasiado lento" 13 .

En Panamá, Rolando Miguel Armuelles Velarde también se queja por las lentas conexiones a Internet. "Nuestro ancho de banda es muy pequeño; como consecuencia, la conexión es lenta y esto frustra a la mayoría de nuestros usuarios. En este momento estamos negociando un ancho de banda de $64 \mathrm{Kbps}$, el que nos pondrá seguramente en el mapa de Internet". Sin embargo, éste no es el único problema que él ve en Panamá: "Es difícil obtener libros actualizados sobre Internet, lo que significa que tenemos que aprender en la $W W W$. Esto toma demasiado tiempo. Es difícil obtener recursos para mejorar, pero cada vez las autoridades de la USMA [Universidad Santamaría La Antigual descubren el potencial que existe en Internet, y esto es bueno para nosotros. El mayor desafío es llevar la cultura de la información a estudiantes y a profesores que no están acostumbrados a ella y que le temen a los computadores" 14

En Guatemala, como se dijo más arriba, el gobierno está tratando de controlar el tipo de información existente para los ciudadanos del país. Otro desafío, según Fernando Behar, de la compañía Behar Productions, es la educación. Lo resume en tres puntos: (a) La gente no sabe lo que es Internet. (b) El costo de enseñar lo que es Internet y sus beneficios es muy alto, porque hay que invertir mucho tiempo es estas actividades. (c) El mayor desafío para mis negocios, específicamente al hablar sobre publicar páginas Web, es que incluso las empresas más grandes en este país no creen que la publicación las beneficiará."15.

Algunas veces la combinación de problemas económicos y políticos causa fuertes efectos. En junio de 1995, la compañía estadounidense Global Enterprise Services desconectó a Venezuela de Internet por cuentas de telecomunicaciones morosas. Durante el escándalo, varios burócratas renunciaron por cargos de corrupción ${ }^{16}$. Aunque los servicios de Internet se reanudaron rápidamente, las empresas se unieron para arreglar sus propias uniones a las redes mundia-
10. LYMAN, ERIC I.:

Peru's catching up with the world; Information means wealth, and Internet provides information", SunSentinel (Fort Lauderdale), 28 de mayo de 1995 p. $21 \mathrm{~A}$.

11. ABasto REVILLA, HUBERT

[hubert@utama.bolnet.bo]: "Re: Preguntas sobre la WWW", email a la autora, 26 de enero de 1996.

12. MENA, YADIRA lyadira@ns.ni]: “Re: Preguntas sobre la WWW", email a la autora, 12 March 1996

13. CORREO DEL WEB [correo@jaguar.uam.rain.ni], "Re: Preguntas sobre la WWW", email a la autora, 14 de marzo de 1996.

14. Armuelles Vel.ARde, ROLANDO MIGUEL. [rolando@darien.usma.ac.pa]: "WWW en Panamá", email a la autora, 26 de marzo de 1996

15. BEHAR, FERNANDO [beharpro@ns.guate.net] "Re: Preguntas sobre la WWW", email a la autora, 15 de marzo de 1996.

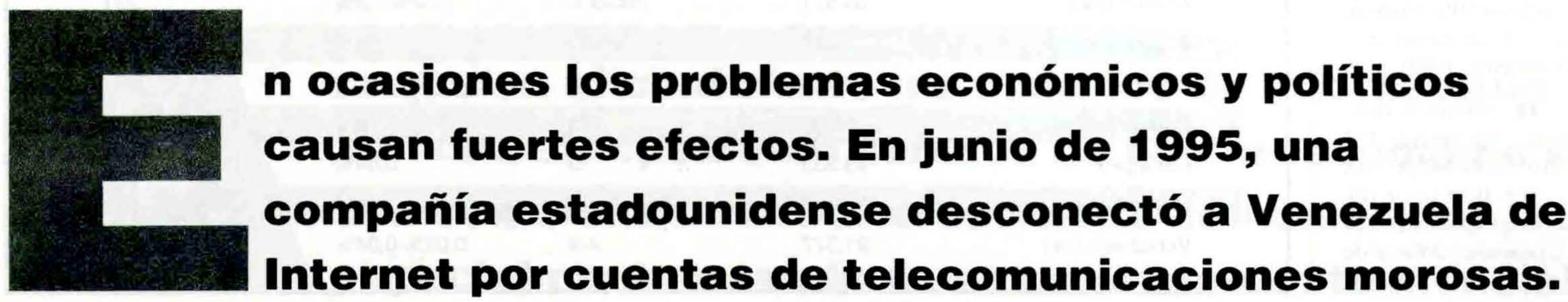


les. Algunas crearon sus propias uniones satelitales cuando no pudieron depender de la infraestructura de las telecomunicaciones de varios países.

Los desafíos que enfrentan los países de América Latina en el desarrollo de Internet fluctúan desde la falta de recursos para la educación y la corrupción política, hasta la información que viene en su gran mayoría en inglés.

Algunos de estos puntos se aclaran cuando se consideran los problemas de acceso a Internet en América Latina.

\section{ACCESO}

La tabla de esta página contiene algunos cálculos y estadísticas relacionados con problemas de acceso a Internet. Proporciona una comparación aproximada de la gente que tiene acceso a la red en varios países. Todas estas cifras son problemáticas y a menudo, muy discutidas. Por ejemplo, en los Estados Unidos estudios citan cifras de acceso de entre un 3,6 y un 17\%. Obviamente, el número de gente que tiene acceso a Internet con frecuencia no es mayor que una estimación (los cálculos varían de persona a persona). Como escribió un webmaster en Venezuela, "lo que usted puede conocer con seguridad científica son los bultos, no a la gente "17. Para su estimación, el Dr. Juan Rivero calculó el número de computadores conectados a Internet en varias universidades e instituciones
16. CARSON, ROY $\mathrm{S}$ : "Venezuela booted from Internet for unpaid phone bills", The American Reporter; 7 de agosto de 1995 .

17. Rivero, JuAN: op. cit.

18. Statistical Yearbook for Latin America and the Caribbean, 1994 edition (Chile: Economic Commission for Latin America and the Caribbean, United Nations, 1995), p. 173;

U.S. BUREAU OF THE Census: Statistical Abstract of the United States: 1995, 115th edition (Washington.

D.C.: U.S. Department of Commerce, 1995), p. 8.

19. Statistical Yearbook... op. cit., pp. 173 y $210-211$ (1980 prices);

U.S. BUREAU OF THE Crnsus: op. cit., p. 456 (constante: dólares de 1987).

\section{ACCESO COMPARATIVO A INTERNET}

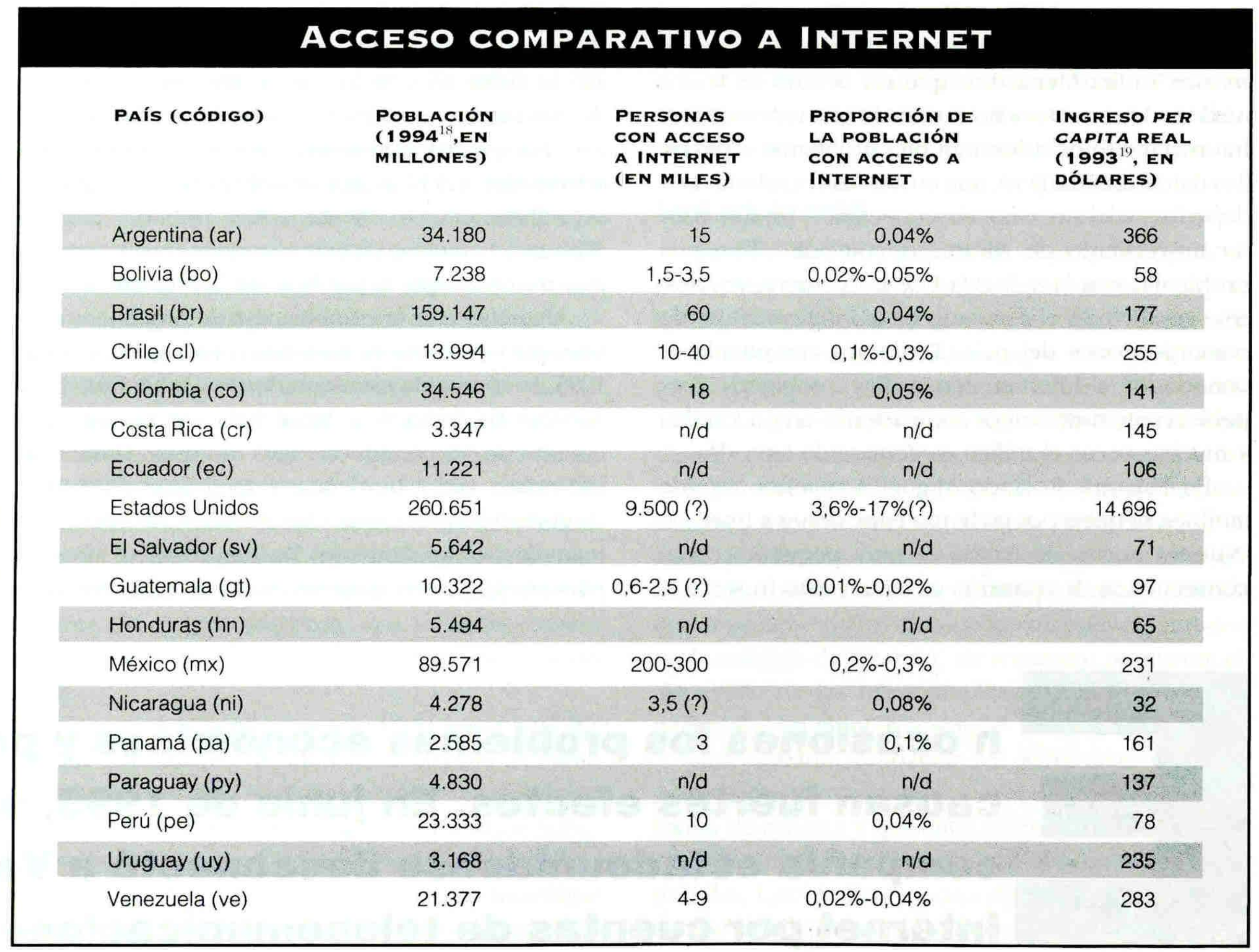


venezolanas y luego los multiplicó por un número aproximado de personas por computador (cuatro). La tabla $\mathrm{N}^{\circ} 1$ también muestra una lista del ingreso disponible nacional bruto percapita en varios países. Este indicador también es problemático por una gran cantidad de razones. Por ejemplo, no incluye factores relacionados con la distribución de la riqueza.

A pesar de los problemas con las cifras en la tabla, los números permiten comprender los desafíos que supone el acceso a Internet en América Latina. El más obvio es una cuestión práctica de quién realmente puede permitirse comprar el hardware necesario para conectarse. Los cálculos indican que el uso de Internet en América Latina no estará basado en individuos que tienen computadores personales y modems en sus hogares, sino más bien en el acceso grupal ligado principalmente a empresas comerciales, universidades, gobiernos y otras instituciones con recursos para invertir en hardware. Esto es evidente en la manera en que se establece el servicio Internet en los países de América Latina. Sólo los costos asociados con Internet contribuirían a poner el acceso en las manos de la elite en varios países. Numerosos estudios sobre usuarios de Internet, cuyos perfiles coinciden con los de los dueños de computadores, en los Estados Unidos y Canadá, indicaron que el típico usuario de la red es "un blanco extremadamente atractivo (educado, profesional y de estratos altos)" para los negocios ${ }^{20}$. La mayoría de estos usuarios son varones, pero, según los estudios, el número de mujeres crece rápidamente. En México, la mayor parte de los usuarios todavía son estudiantes universitarios y la otra parte es un grupo reducido de habla inglesa. Aunque en América Latina los usuarios de Internet aún son una elite, existen algunas señales de que la tecnología se usa como una forma alternativa de comunicación, especialmente cuando se observa en un contexto mundial.

\section{UNA "VENTANA AL MUNDO"}

Juan Carlos Cabrera Portillo, ingeniero del Instituto de Nutrición de Centroamérica y Panamá, indica que la finalidad de sus páginas Web fue crear una "ventana al mundo" donde ellos podrían presentar información acerca de sus tareas diarias, así como también objetivos y éxitos del Instituto ${ }^{21}$. En América Latina Internet es usado de muchas formas por diferentes organizaciones. En esta sección, me centraré principalmente en usos no comerciales de las comunicaciones Internet.

En los últimos años, Internet se ha convertido en una herramienta esencial en los conflictos políticos y sociales. Por ejemplo, pocos días antes de que estallara la crisis limítrofe entre Perú y Ecuador en 1995 , comenzó una "guerra electrónica". Ecuador publicó información crítica sobre Perú en Internet, supuestamente utilizando textos de Sendero Luminoso; y Perú se vengó con información de su propiedad. Este país aún combate a ese grupo terrorista, pero ahora en el ciberespacio. Ambas partes, al usar el mismo servidor en la ciudad de Nueva York están tratando de identificar las ubicaciones de los computadores de cada uno en Perú y cuando se identifiquen, borrar todos los datos ${ }^{22}$.

Casi una década antes, el Frente de Liberación Nacional Farabundo Martí de El Salvador también usó Internet en su lucha contra el gobierno.

Quizás el uso más publicitado de Internet en un conflicto fue el de los zapatistas en el estado sureño de Chiapas, en México. Al caso, se lo ha llamado una
20. CommerceNet Nielsen Internet Demographics Survey, Executive Summary, [http://

www.nielsenmedia.com whatsnew/ execsum2.htm], 1995.

21. Cabrera portillo, Juan Carlos [jcabrera@ns.incap.org.g]: "Re: Preguntas sobre la WWW", email a la autora, 12 de marzo de 1996.

22. "Flash point 10 : Peru-from guerrilla warfare to warfare computer", Computer Fraud \& Security Bulletin, Elsevier Advanced Technology Publications, 1 de enero de 1996.

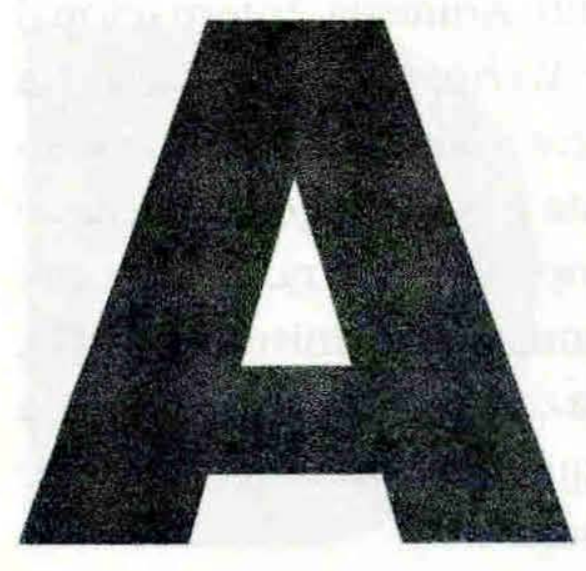

unque en el continente los usuarios de Internet aún son una elite, existen señales de que la tecnología se usa como una forma alternativa de comunicación, especialmente cuando se ve en un contexto mundial. 
23. LANGFIELD, MARTIN: "Feature-Mexican rebels take case to cyberspace, Reuters, 14 de diciembre de 1995 .

24. "Marcos on the Internet", The Christian Science Monitor, 27 de febrero de 1995 , p. 20. "guerra de Internet", la "guera en red prototipo del siglo veintiuno", una "guerra de/ información", "rebelión de la era de la información y una "guerra de propaganda". Desde que el conflicto estalló en 1994, los zapatistas (Ejército Zapatista de Liberación Nacional o EZLN) han usado Internet para presionar al gobierno mexicano, para dar publicidad a su cau-

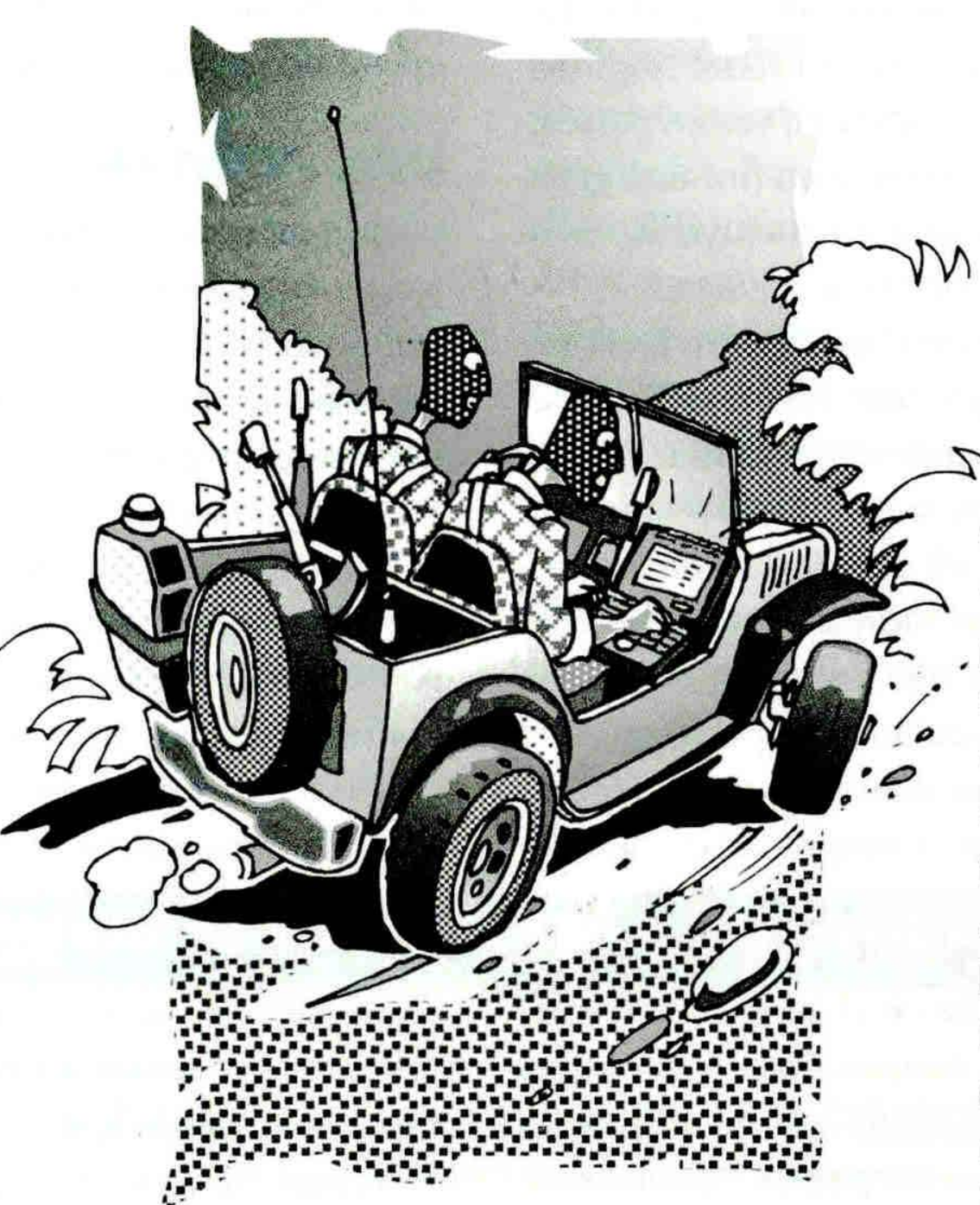

últimos datos acerca de las operaciones militares y le piden a la gente de todo el mundo inundar la oficina del Presidente mexicano con mensajes de protesta a través del teléfono y del fax. Ambas partes aseguran que la información en Internet ha sido falseada, como los reportes de breves ejecuciones generales y de torturas brutales por parte del ejércisa y ganar apoyo internacional. El uso de Internet está cambiando la naturaleza de la guerrilla y de la protesta cívica; porque, a pesar de que ha habido tiroteos en los dos años desde que comenzó el levantamiento, los enfrentamientos se han dado principalmente a punta de gritos de información a través de Internet. El subcomandante Marcos lo resumió así: "Lo que los gobiernos realmente deberían temer es a un experto en comunicaciones".

Los mensajes de los zapatistas se envían a los grupos de derechos humanos alrededor del mundo, incluyendo PeaceNet, Chiapas-List, Mexpaz, La Neta y México 94. De ahí, la información es recolectada por activistas y por nuevas organizaciones, quienes luego publican los comunicados. Harry Cleaver, un profesor de la Universidad de Texas en Austin, que mantiene una página Web sobre Chiapas, indica que Internet permite a los zapatistas liberarse "ellos mismos de represiones geográficas. Ni siquiera pueden viajar a Ciudad de México para dar discursos, pero ésta es una forma de estar en Texas, Roma, California o París ${ }^{23}$. Los partidarios del EZLN informan los to mexicano. "El fenómeno Marcos-Internet muestra cómo México está siendo atado más firmemente a la comunidad mundial por medio de demandas que se adecuan a los modelos internacionales de los derechos humanos, que por los esfuerzos de tratados como el de Libre Comercio de América del Norte o como las garantías de prestamos internacionales)”24. A pesar de que los zapatistas últimamente han estado perdiendo su perfil de grandes usuarios de los medios de comunicación, continúan vigilando la situación a través de Internet.

El EZLN no es el único grupo en América Latina que utiliza Internet para sus propósitos. En todo el mundo, asociaciones como Amnistía Internacional usan los sistemas Internet y Webpara comunicarse. En El Salvador, Amnistía recurre a Internet para recolectar información rápidamente y para luego contactarse con las autoridades apropiadas cuando sabe de abusos a los derechos humanos. Al mismo tiempo, obtiene la información para entregarla a los grupos de presión internacionales, quienes pueden aunar su "experiencia de nivel mundial". 
Activistas brasileños y grupos civiles sin fines de lucro usan Internet desde hace ocho años, aproximadamente. En 1988, cuando asesinaron a Chico Mendes, el seringueiro que defendía los bosques tropicales en el Amazonas, la noticia se difundió a través Internet. Activistas sociales usan computadores y modems en las luchas por los pobres, por las minorías, por las mujeres y por el medio ambiente; organizaciones no gubernamentales están conectadas unas con otras y con Internet; pueden publicar las amenazas de muerte contra los campesinos sin tierras por parte de grandes terratenientes en el estado norte de Tocantis, enviar estadísticas de deforestación a las oficinas de Greenpeace o antecedentes sobre la conservación de la vida silvestre.

El movimiento ambiental peruano ha crecido en los últimos cinco años, pero permanece concentrado principalmente en las clases media y alta. Los grupos, muchos de ellos ONGs, usan el correo electrónico para comunicarse entre ellos y con otras organizaciones. Por ejemplo, coordinan protestas contra los ensayos nucleares franceses en el sur del Pacífico. En México, activistas ambientales también se comunican a través de Internet. En un incidente, un mexicano que estaba en Greenlink, la red computacional para Greenpeace, se unió con oficinas en Gran Bretaña para protestar contra el envío de desechos tóxicos a su país. Joe Keenan, director del programa de Conservación de la Naturaleza del sur de México, habla del futuro de una red de grupos ambientales conectados a Internet en ese país, Guatemala y Belice: "Reunirá a todos estos grupos que ahora están muy diseminados (ellos pierden la oportunidad de beneficiarse de lo que sus colegas hacen, incluso a cien kilómetros de distancia, ya que no cuentan con un presupuesto para viajes). Entrar en un servicio de red le permitirá a un grupo hacer una investigación sobre el árbol mangle en las costas del Pacífico y comparar inmediatamente notas con sus colegas en el Golfon.

"Los pocos grupos mexicanos sin fines de lucro que usan comunicación electrónica han encontrado que los trabajos en red los ayudan a participar de una manera más eficiente en la toma de decisiones sobre política pública", asegura Jacques Lefeyre, quien dirige La Neta, una red en la Ciudad de México que prepara a grupos que trabajan en materias de medio ambiente, derechos humanos, desarrollo y paz. En 1991, un grupo de ONGs mexicanas (Casa y Ciudad, Centro Operacional de Vivienda, Grupo de Estudios Ambientales, Mujer a Mujer, Servicios Informativos Procesados) comenzó a crear uniones de redes computacionales. Pidieron prestado un computador y compraron una línea telefónica y un modem para lanzar un sistema de boletines. Lefeyre recuerda: "A pesar de que sus capacidades eran limitadas, este primer sistema permitió a las organizaciones empezar a usar esta tecnología para comunicarse con otras en el resto del país, para anunciar acontecimientos próximos, para denunciar violaciones a los derechos humanos, promover la educación y discutir asuntos relacionados con el Nafta, el medio ambiente, la igualdad de los sexos y sucesos mexicanos de actualidad. Hemos creado una comunidad sin límites con redes que crean un amplio foro internacional 25 .

Los ecuatorianos que trabajan en el extranjero se mantienen al tanto de los acontecimientos actuales a través de un boletín de noticias en línea en EcuaNet ${ }^{26}$. Los chilenos que viven en otros países han adoptado las publicaciones en línea de diarios y revistas chilenos, como La Época y El Mercurio. Miguel Ángel
25. Ibid

26. MANTILLA, JAIME: "Linking the Latins; Further away-but closer together", WorldPaper; mayo de 1995 , p. 4

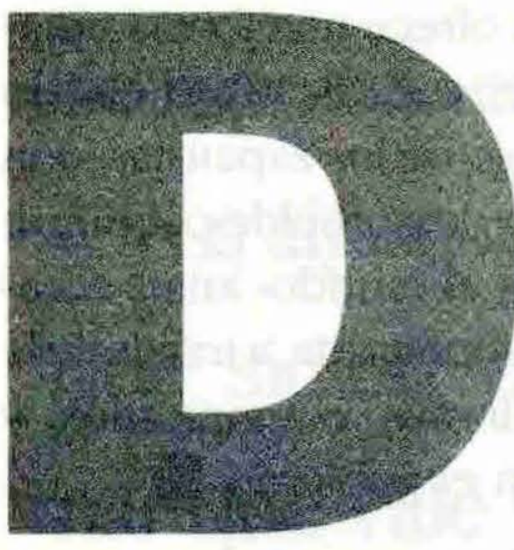

\section{esde que estalló el conflicto de Chipas en 1994, los zapatistas han usado Internet para presionar al gobierno mexicano para dar publicidad a su causa y ganar apoyo internacional.}




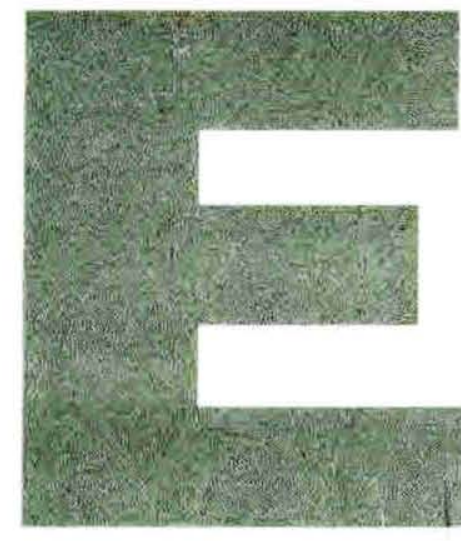

n la mayoría de los países latinoamericanos, al igual que en Estados Unidos, la tendencia parece inclinarse hacia la privatización de las actividades de telecomunicación y al crecimiento de los sistemas de comunicación.

Larrea, editor de La Época Internet, señala que él recibe muchas cartas de chilenos que están ansiosos de leer noticias de su país.

\section{¿GRITOS AL MUNDO?}

"Yo creo en el poder de las comunicaciones Internet", escribe Cristián Espinoza T., quien tiene una página Weben Chile. Por muchos años, dice, se sintió inútil mientras miraba cómo los sucesos acontecían a su alrededor. "Esta vez, tengo la oportunidad de gritar al mundo y siento que alguien me escuchan 27 . Otros usuarios han manifestado sentimientos similares acerca de las posibilidades que Internet proporciona. Algunos participantes en el taller de 1995 sobre la sociedad Internet en los países desarrollados declararon que "una vez que la infraestructura lo permita, las naciones en desarrollo utilizarán los servicios de Internet para sus propios intereses económicos, políticos y sociales". La utilización de Internet por movimientos sociales es "un poco diferente de la que se hace en el primer mundo, donde se hace mucho alarde sobre la habilidad de las redes para poner en el mercado películas y

27. ESPINOZA T., CRISTIÁN (cet@ing.puc.cl): «Re: Preguntas WWW", email a la autora, 17 de noviembre de 1995.

28. HARMON, AMY: "Planting Net seeds in Third World", Los Angeles

Times, 6 de julio de 1995 , p. D1.

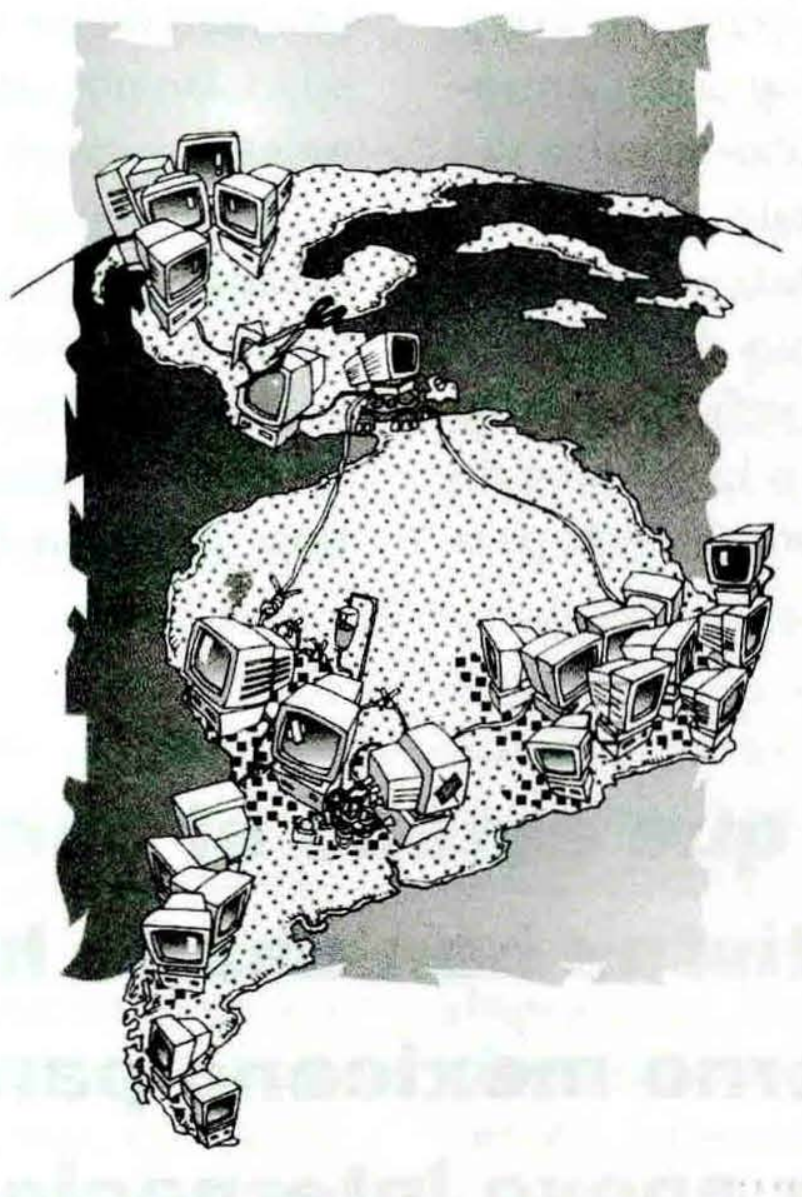

de acceso. Cada nación discute la mejor manera de construir la infraestructura necesaria para las nuevas tecnologías de comunicación. En la mayoría, al igual que en Estados Unidos, la tendencia parece inclinarse hacia la privatización de las actividades de telecomunicación y al crecimiento de los sistemas de comunicación. Las presiones del mercado, la pequeña proporción de gente que tiene acceso, junto con los altos costos y el hecho de que la información se encuentra en gran parte en inglés, aparecen como los causantes de los peores miedos de los críticos interesados en comunicaciones más democráticas.

Sin embargo, el uso de Internet por varios grupos en América Latina parecería indicar posibilidades para formas alternativas de comunicación. Además, en algunos países, como Chile, los costos de conexión están bajando aceleradamente, con lo que el grupo de personas con acceso a Internet se está ampliando también muy rápido. En toda América Latina, distintos grupos y movimientos sociales usan Internet para fortalecer los lazos locales e internacionales. Utilizan la red para ganar apoyo para sus causas y para diseminar información. Soledad Ferreiro, de la Universidad de Chile, afirma que Internet ofrece una forma notable de poder para la gente que lo usa. En el caso de los zapatistas, con la tecnología los rebeldes abrieron una "ventana al mundo" antes completamente impensada, a través de la cual difundir sus comunicados y negociar con el gobierno.Cl 